\title{
Color-magnetic flux tubes in quark matter cores of neutron stars
}

\author{
Mark G. Alford* \\ Physics Department, Washington University, St. Louis, MO 63130-4899, USA \\ Armen Sedrakian ${ }^{\dagger}$ \\ Institute for Theoretical Physics, J. W. Goethe-University, \\ D-60438 Frankfurt am Main, Germany
}

(Dated: 10 Feb 2010)

\begin{abstract}
We argue that if color-superconducting quark matter exists in the core of a neutron star, it may contain a high density of flux tubes, carrying flux that is mostly color-magnetic, with a small admixture of ordinary magnetic flux. We focus on the two-flavor color-superconducting ("2SC") phase, and assume that the flux tubes are energetically stable, although this has not yet been demonstrated. The density of flux tubes depends on the nature of the transition to the colorsuperconducting phase, and could be within an order of magnitude of the density of magnetic flux tubes that would be found if the core were superconducting nuclear matter. We calculate the crosssection for Aharonov-Bohm scattering of gapless fermions off the flux tubes, and the associated collision time and frictional force on a moving flux tube. We discuss the other forces on the flux tube, and find that if we take in to account only the forces that arise within the 2SC core region then the timescale for expulsion of the color flux tubes from the $2 \mathrm{SC}$ core is of order $10^{10}$ years.
\end{abstract}

*Electronic address: alford@wuphys. wustl.edu

${ }^{\dagger}$ Electronic address: sedrakian@th.physik.uni-frankfurt.de 


\section{INTRODUCTION}

It has long been conjectured that neutron stars might contain cores of quark matter, and one of the challenges facing nuclear astrophysics is to find signatures by which the presence of such matter could be inferred from observations of the behavior of neutron stars. This requires us to develop a good understanding of the differences between the properties of nuclear matter and quark matter, taking in to account the effects of magnetic fields, which are known to be present in neutron stars. In this paper we study quark matter in magnetic fields $B \lesssim 10^{14}$ Gauss, which are astrophysically plausible and high enough to affect transport (see for example [1]) but not so large as to modify the phase structure of the material [2-4].

Nuclear matter at high densities and low temperatures is expected to be a type-II electrical superconductor, with the magnetic field distributed in an Abrikosov lattice of flux tubes [5]. In this paper we investigate the possibility that quark matter in the two-flavor color superconducting phase ("2SC") [6] could be a type-II superconductor with respect to the color gauge fields [7,8], with color flux tubes that scatter electrons, muons, and ungapped quarks via the Aharonov-Bohm effect. These tubes are not topologically stable, and their energetic stability has not yet been determined; in this paper we investigate the role they might play in transport, and their expulsion time, if they turn out to be stable or to have a lifetime that is sufficiently long. As we explain below, the tubes carry flux that is mostly color-magnetic (hence they can reasonably be called "color-magnetic flux tubes") with a small admixture of ordinary magnetic flux. We will argue that the density of colormagnetic flux tubes could be high, perhaps only about an order of magnitude less than that of ordinary flux tubes in superconducting nuclear matter. Color magnetic flux tubes may appear in other color superconducting phases, such as the color-flavor-locked (CFL) phase, but the CFL phase has no gapless charged excitations, so in this paper we focus on the 2SC phase. We calculate the Aharonov-Bohm interaction between the flux tubes and unpaired quarks or electrons/muons. We calculate the associated damping time and the forces on the flux tubes. We defer the calculation of other contributions to relaxation and transport in the 2SC phase, such as scattering of the unpaired quarks and electrons off each other, to future work.

The behavior of quark matter phases in magnetic fields is complicated by the intertwined breaking of the strong interaction $S U(3)$ "color" gauge symmetry and the electromagnetic $U(1)_{Q}$ gauge symmetry. In the $2 \mathrm{SC}$ phase, a condensate of Cooper pairs of up $(u)$ and down $(d)$ quarks leads to the gauge symmetry breaking pattern $S U(3) \otimes U(1)_{Q} \rightarrow S U(2)_{r g} \otimes U(1)_{\tilde{Q}}$ $[9,10]$. The unbroken $S U(2)_{r g}$ symmetry ensures confinement of particles that carry net red or green color, with a confinement scale around $10 \mathrm{MeV}$ [11]. The unbroken $U(1)_{\tilde{Q}}$ gauge symmetry is a linear combination of the original electromagnetic and color symmetries, called "rotated electromagnetism". The associated gauge field, the "Q $\tilde{Q}$ photon", is a combination of the original photon and one of the gluons. It is massless and propagates freely in 2SC quark matter. The orthogonal combination $X$ is a broken gauge generator, and the associated magnetic field has a finite penetration depth.

The situation is closely analogous to the Higgs mechanism in the standard model, where one linear combination of the hypercharge and $W_{3}$ gauge bosons remains massless (the photon), while the orthogonal combination becomes massive (the $Z^{0}$ ). The $X$ flux tubes are therefore analogous to " $Z$-strings" [12] which have been found to be stable only in a small region of the standard model parameter space [13], although the stable region may 
be enlarged when bound states are taken in to account [14]. There are differences between the 2SC phase of QCD and the Higgs phase of the standard model: the gluon mass is proportional to the quark chemical potential, not the superconducting order parameter [6]; the non-Abelian gauge group is $S U(3)$ rather than $S U(2)$ and is only partly broken, leaving an unbroken confining $S U(2)$ as well as an unbroken $U(1)$ in the low temperature phase. This means that a separate stability calculation will be needed for the $2 \mathrm{SC}$ case.

Because electromagnetism is much more weakly coupled than the strong interaction, the massless $\tilde{Q}$ gauge field is almost identical to the photon, with a small admixture of a color gauge boson. Conversely, the broken $X$ gauge field is almost identical to one of the gluons, with a small admixture of the photon [10]. Thus the $X$ flux tubes can be described as "color-magnetic flux tubes". However, because they contain a small admixture of ordinary magnetic flux, they interact with electrons/muons as well as with unpaired (blue) quarks. In summary, the 2SC phase is not a superfluid, but it is a superconductor with respect to the $X$ gauge fields, and a conductor with respect to the $\tilde{Q}$ gauge fields, with current mainly being carried by the gapless electrons and blue quarks (one of which is neutral, the other has charge +1 ). Strange quarks and muons, if present, will have a lower Fermi momentum because of their higher mass, and hence less phase space near their Fermi surface. Thus their contribution to the processes discussed in this paper will be subleading, and we ignore it.

The picture given above is valid below the critical temperature for $2 \mathrm{SC}$ pairing and above an unknown critical temperature $T_{1 S C}$ at which there will be a transition to a phase in which there is self-pairing of the blue up and down quarks. Such pairing would break the $U(1)_{\tilde{Q}}$ symmetry, so there could be both $\tilde{Q}$ and $X$ flux tubes. Models of the strong interaction between quarks do not give us much idea of the value of $T_{1 S C}$. They agree that, because the strong attraction is much weaker in the single-color channel, $T_{1 S C}$ will be many orders of magnitude lower than the critical temperature for $2 \mathrm{SC}$ pairing, perhaps as low as $1 \mathrm{eV}$ $\left(10^{4} \mathrm{~K}\right)[9,15,16]$. In this paper we will be concerned with temepratures above $T_{1 S C}$, where the $\tilde{Q}$ gauge symmetry remains unbroken.

Depending on the ratio of the $X$-flux penetration depth to the coherence length of the condensate, the $2 \mathrm{SC}$ phase may be type-I or type-II with respect to the $X$ magnetic field [7]. In this paper we will be concerned with the possibility of type-II behavior, and the presence of flux tubes containing $X$-flux in the $2 \mathrm{SC}$ quark matter core of a compact star. Even if the average magnetic field strength in the core is below the lower critical field, such flux tubes may end up "frozen in" if the quark matter had cooled in to the 2SC state in the presence of the magnetic field. The magnetic field would then be resolved in to a $\tilde{Q}$ part, which would pass freely through the 2SC quark matter, and an $X$ part, which would become trapped in flux tubes (Sec. III).

The paper is structured as follows. In Sec. II we calculate the Ginzburg-Landau parameter for 2SC quark matter, and conclude that it is a type-II superconductor with respect to the broken $X$ generator as long as the pairing gap $\Delta$ is large enough. We estimate that $\Delta \gtrsim \mu_{q} / 16$ will suffice, which for typical quark chemical potentials $\mu_{q} \sim 400 \mathrm{MeV}$ requires $\Delta \gtrsim 25 \mathrm{MeV}$. In Sec. III we discuss the nucleation scenario by which the flux tubes can occur in the 2SC superconductor, even when the magnetic field intensities are below the lower critical field. We estimate the density of such flux tubes in the hypothetical 2SC quark matter core of a neutron star. In Sec. IV we calculate the Aharonov-Bohm scattering cross section for electrons or unpaired quarks interacting with color magnetic flux tubes. Sec. V is devoted to the computation of relaxation time of massless electrons and unpaired blue 
quarks interacting with flux tubes via Aharonov-Bohm cross-section. In Sec. VI we estimate the timescale for expulsion of the flux tubes from the 2SC core, taking in to account the forces on the color-magnetic flux tubes in the 2SC core and at its boundary, but neglecting any forces on the magnetic flux lines outside the core. We summarize our results in Sec. VII.

In our calculations we use "Heaviside-Lorentz" natural units with $\hbar=c=k_{B}=\epsilon_{0}=1$, where $k_{B}$ is the Boltzmann constant and $\epsilon_{0}$ is the vacuum permittivity; the electric charge $e$ is related to the fine structure constant by $\alpha=e^{2} /(4 \pi)$.

\section{TYPE-II COLOR SUPERCONDUCTIVITY IN QUARK MATTER}

A superconductor is of type II if it obeys the condition

$$
\kappa \equiv \frac{\lambda}{\xi}>\frac{1}{\sqrt{2}}
$$

where $\kappa$ is the Ginzburg-Landau (GL) parameter, $\lambda$ is the penetration depth, and $\xi$ is the coherence length for the superconductor. In a system of relativistic fermions with chemical potential $\mu$ and pairing gap $\Delta$, we expect $\xi \propto 1 / \Delta, \lambda \propto(g \mu)^{-1}$, so $\kappa \propto \Delta /(g \mu)$. (In the case of $2 \mathrm{SC}$ quark matter the relevant broken gauge symmetry is the " $X$ " which is mostly color, so the coupling $g$ is approximately the strong coupling constant.) We therefore expect that 2SC quark matter will be a type-II color superconductor if the gap is sufficiently large.

To make a more accurate determination we follow the approach of Bailin and Love [17] and Iida and Baym [7]. We start with the effective free energy density (Ginzburg-Landau theory) for a relativistic BCS superconductor (Ref. [17], (3.12))

$$
\mathcal{F}=\mathcal{F}_{n}+\alpha \psi^{*} \psi+\frac{1}{2} \beta\left(\psi^{*} \psi\right)^{2}+\gamma\left(\nabla \psi^{*}-2 i e \boldsymbol{A} \psi^{*}\right)(\nabla \psi+2 i e \boldsymbol{A} \psi)+\frac{1}{2 \mu_{0}}\left(\boldsymbol{B}-\mu_{0} \boldsymbol{H}\right)^{2}
$$

(We have followed Ref. [17] in writing the magnetic field free energy in SI units; in natural units $\mu_{0}=1$.) Here $\psi$ is the gap parameter; for negative $\alpha$ the free energy has a minimum at $|\psi|^{2}=\psi_{0}^{2}$, with penetration depth $\lambda$, and coherence length $\xi$ given by

$$
\psi_{0}^{2}=-\frac{\alpha}{\beta}, \quad \lambda^{2}=\frac{1}{2 \gamma q_{\text {pair }}^{2}\left|\psi_{0}\right|^{2}}, \quad \xi^{2}=-\frac{\gamma}{\alpha},
$$

where $q_{\text {pair }}$ is the charge of the Cooper pair. The GL parameter $\kappa$ is then given by

$$
\kappa^{2}=\frac{\lambda^{2}}{\xi^{2}}=\frac{1}{2 q_{\text {pair }}^{2}} \frac{\beta}{\gamma^{2}} .
$$

The coefficients in the Ginzburg-Landau functional are [17]

$$
\begin{aligned}
\alpha & =\nu \frac{\tau_{G L}}{2} \\
\beta & =\nu \frac{7 \zeta(3)}{16\left(\pi T_{c}\right)^{2}}, \\
\gamma & =\frac{\beta}{6} \frac{p_{F}^{2}}{\mu^{2}}
\end{aligned}
$$


where $\tau_{G L} \equiv\left(T-T_{c}\right) / T_{c}$. The fermions have Fermi momentum $p_{F}$, so the density of states near the Fermi surface is $\nu=N p_{F} \mu / \pi^{2} \simeq N \mu^{2} / \pi^{2}$. The parameter $N$ is a degeneracy factor that is 1 for a single-species system, and 2 for the 2SC phase (see Ref. [17], Eq. (4.63)). The Ginzburg-Landau theory is most reliable for temperatures close to $T_{c}$, however we will use it at $T \ll T_{c}$. The low-temperature gap parameter $\Delta$ is related to the critical temperature by $T_{c}=\left(e^{\gamma} / \pi\right) \Delta$ : note that $\Delta$ then differs from $\psi_{0}$ by a factor of about 1.7 . Expressing the coefficients in terms of $\Delta$,

$$
\kappa \approx \frac{32.74}{q_{\text {pair }} \sqrt{N}} \frac{\Delta}{\mu}, .
$$

We can check this result by noting that for a relativistic electronic superconductor, $N=1$ and $q_{\text {pair }}=2 e$ where $\alpha=e^{2} /(4 \pi) \approx 1 / 137$. Substituting these values into (6) we find $\kappa=54.043 \Delta / \mu=95.325 T_{c} / \mu$, in agreement with Ref. [17], (3.24).

In $2 \mathrm{SC}$ quark matter, the degeneracy factor is $N=2$ and the charge of the Cooper pair is the $X$ charge of the 2SC condensate. From Eqs. (29) and (31) of Sec. IV we find

$$
q_{\text {pair }}=q_{c} e^{(X)}=\frac{g}{\sqrt{3} \cos \varphi} \approx \frac{g}{\sqrt{3}},
$$

where the mixing angle $\varphi$ is defined in Eq. (29). We estimate the strong coupling constant $g$ by assuming that $\alpha_{s}=g^{2} /(4 \pi) \approx 1$, so $g \approx 3.5$. Substituting these values into (6) we find

$$
\kappa_{2 \mathrm{SC}} \approx 11 \frac{\Delta}{\mu_{q}}
$$

We conclude, using (1), that 2SC quark matter will be of type II if the pairing gap is sufficiently large, $\Delta \gtrsim \mu_{q} / 16$. In quark matter we expect $\mu_{q} \sim 400 \mathrm{MeV}$, so this only requires the $2 \mathrm{SC}$ pairing gap to be greater than about $25 \mathrm{MeV}$, which is well within typical estimates $[18,19]$. Our general conclusion agrees with that of Refs. [7, 8] who also noted that a sufficiently large 2SC pairing gap yields a type-II superconductor. Our specific result (8) differs from Eq. (112) of Ref. [7] by a factor of $\sqrt{2}$, but given the uncertainty in the strong coupling constant $g$ this numerical discrepancy does not affect our conclusion.

\section{COLOR-MAGNETIC FLUX TUBES IN THE 2SC PHASE}

\section{A. The nucleation and density of flux tubes}

When the quark matter core of the star cools below a critical value, a 2SC condensate forms. We expect that this happens before the nuclear mantle becomes superconducting because the gap parameter for quark matter is expected to be an order of magnitude larger than that for proton pairing [6,20-22]. The electromagnetic field is then resolved in to a $\tilde{Q}$ component and an $X$ component. The $2 \mathrm{SC}$ core is not a superconductor with respect to $\tilde{Q}$, so the $\tilde{Q}$ component is undisturbed [10] (on this we disagree with Ref. [23], which we believe imposes an incorrect boundary condition on the gluon field). However, the core is a superconductor with respect to the $X$ component, and we have argued above that it may well be a type-II superconductor. The lower critical field for the $X$-superconductivity is very high, $H_{c 1} \sim 10^{17}$ Gauss [7], and typical neutron star magnetic fields are expected to be lower than this, but, as we now argue (see also [10] and footnote [8] of Ref. [7]), it is still 
quite possible for the $X$-flux to form flux tubes threading the quark matter core. The only way the $X$-flux could be expelled from the core is if the transition from hot quark matter to 2SC happens smoothly from the center of the star outwards. However, it seems more likely that the transition to 2SC matter will proceed by nucleation of 2SC regions ("bubbles") in the quark matter, which then grow and coalesce. The $X$-flux will be expelled from the 2SC bubbles, but will then be trapped in the non-superconducting regions between the bubbles. As the bubbles grow, these regions become smaller, concentrating the flux there until the local field strength rises above $H_{c 1}$, at which point the bubbles stop growing. At this stage, the core consists of 2SC quark matter with channels of non-superconducting quark matter running though it, carrying the $X$-flux. If the 2SC phase is a type-II superconductor then these channels are unstable and will fragment into flux tubes, each carrying a single quantum of $X$-flux, with a short-range repulsion between the flux tubes. The fact that the average field strength was below the lower critical field for a sphere of $2 \mathrm{SC}$ matter in a uniform magnetic field will now manifest itself as an outwardly-directed boundary force on the flux tubes at the point where they meet the edge of the 2SC core. We will study this in Sec. VI.

Because the 2SC phase is a conductor with respect to $\tilde{Q}$ charge, it supports eddy currents which make it very difficult for the $\tilde{Q}$ magnetic field in the $2 \mathrm{SC}$ core to change. The timescale for expulsion of the $\tilde{Q}$ magnetic field is estimated to be longer than the age of the universe [10]. Thus we are justified in treating the $\tilde{Q}$ magnetic field as a fixed background.

If we assume that all the $X$-flux is trapped in the manner described above, then the density of flux tubes is just $B_{X}$, the density of magnetic $X$-flux, divided by $\Phi_{X}$, the $X$-flux of a single flux tube. $B_{X}$ is obtained by projecting out the $X$-component of the original electromagnetic flux $B$ (see $(27)$ ), so $B_{X}=B \sin \varphi$. The flux quantum is

$$
\Phi_{X}=\frac{2 \pi}{q_{\text {pair }}} \approx \sqrt{\frac{3 \pi}{\alpha_{s}}}
$$

where $q_{\text {pair }}$ is the $X$-charge of the $2 \mathrm{SC}$ condensate (see (7) and (29)). We can relate it to the flux quantum $\Phi_{0}=\pi / e \approx 10.37$ for an ordinary superconductor where the charge of the condensate is $2 e$,

$$
\Phi_{X}=\frac{2 e}{q_{\text {pair }}} \Phi_{0}=6 \sin (\varphi) \Phi_{0}
$$

We conclude that

$$
n_{v}=\frac{B_{X}}{\Phi_{X}}=\frac{1}{6} \frac{B}{\Phi_{0}} .
$$

This is the upper limit on the flux tube density in 2SC matter. Interestingly, as anticipated in Ref. [24], it only differs by a factor of $1 / 6$ from the density of electromagnetic flux tubes that would result if the core were an electromagnetic superconductor due to electron or proton pairing. Projection on to the $X$ component reduces the magnetic flux by a factor $\sin \varphi$, but because the $X$ fields are strongly coupled their flux quantum is smaller by a similar factor, so the flux tube density ends up being independent of the mixing angle. The actual density will depend on details of how the transition to 2SC matter was completed. For an internal field $B=10^{14}$ Gauss $\left(2 \mathrm{MeV}^{2}\right)$, the maximum flux tube density is $n_{v}=8.1 \times 10^{19} \mathrm{~cm}^{-2}$.

\section{B. Properties of the flux tube}

The thickness of the flux tubes is given by the penetration depth for magnetic $X$-flux in the 2SC phase. This follows from equations (4) to (7). Assuming $p_{F} \simeq \mu_{q}$ for relativistic 
quarks,

$$
\lambda=\frac{3 \pi}{g \mu_{q}\left|\tau_{G L}\right|^{1 / 2}}=(1.3 \mathrm{fm})\left(\frac{400 \mathrm{MeV}}{\mu_{q}}\right)\left(1-\frac{T}{T_{c}}\right)^{-1 / 2} .
$$

The energy per unit length (tension) of the flux tube is given by $\frac{1}{2} \mathcal{E} \ln \kappa_{X}$ where $\mathcal{E}$ is the energy per unit length of the magnetic flux if it were uniformly spread over an circle of radius $\lambda$ (Ref. [25], Sec. (5.1.2)), and $\ln \kappa_{X}$ is a factor of order 1. In Heaviside-Lorentz natural units $\mathcal{E}=B^{2} / 2$, where $B=\Phi_{X} /\left(\pi \lambda^{2}\right)$, so

$$
\varepsilon_{X}=\frac{\Phi_{X}^{2}}{4 \pi \lambda^{2}} \ln \kappa_{X}
$$

(compare Ref. [7], Eq. (107); see also Ref. [24]). To estimate the tension we work to lowest order in $\alpha$ and use (12), (9), and (34). In the low temperature limit we find

$$
\varepsilon_{X}=\frac{\mu_{q}^{2}}{3 \pi} \ln \kappa_{X}
$$

Assuming that in 2SC quark matter $\mu_{q}$ is in the 350 to $500 \mathrm{MeV}$ range, and that the logarithmic factor is of order 1 , we conclude that the tension will be of order 60 to $130 \mathrm{MeV} / \mathrm{fm}$.

\section{AHARONOV-BOHM SCATTERING BY FLUX TUBES}

The Aharonov-Bohm effect provides a remarkably strong interaction between a charged particle and a flux tube containing magnetic flux. For the simple case of a single $U(1)$ gauge group (electromagnetism), the differential cross-section per unit length is (see, for example, Ref. [26])

$$
\frac{d \sigma}{d \vartheta}=\frac{\sin ^{2}(\pi \tilde{\beta})}{2 \pi k \sin ^{2}(\vartheta / 2)}
$$

where

$$
\tilde{\beta}=\frac{q_{p}}{q_{c}}
$$

where $q_{p}$ is the charge of the scattering particle. For a flux tube that arises as a topological soliton in an Abelian Higgs model, $q_{c}$ is the charge of the condensate field whose winding by a phase of $2 \pi$ characterizes the flux tube; $k$ is the momentum in the plane perpendicular to the string, and $\vartheta$ is the scattering angle. Aharonov-Bohm scattering has several important features:

- The cross-section vanishes if $\tilde{\beta}$ is an integer, but is otherwise non-zero.

- The cross section is independent of the thickness of the flux tube: the scattering is not suppressed in the limit where the symmetry breaking energy scale goes to infinity, and the flux tube thickness goes to zero.

- The cross section diverges both at low energy and for forward scattering.

It is therefore of great interest to determine the values of $\tilde{\beta}$ for scattering of the fermions that are ungapped in the $2 \mathrm{SC}$ phase off a flux tube containing magnetic flux associated with the broken gauge symmetry. 


\section{A. The gauge groups and charges}

\section{The light fermions}

In the $2 \mathrm{SC}$ phase we will focus on the $U(1) \times U(1)$ gauge group consisting of electromagnetism and the part of the color gauge symmetry that mixes with electromagnetism. The relevant particles are the quarks and the electron:

$$
\psi=\left(r u, g d, r d, g u, b u, b d, e^{-}\right),
$$

where "ru" means the red up quark, etc. " $e^{-"}$ is the electron. Muons would have the same interaction as the electron, so we do not include them separately. In this basis, the generators of the two $U(1)$ gauge groups are just the diagonal matrices of their electric and color charges,

$$
\begin{aligned}
Q^{\psi} & =\operatorname{diag}\left(+\frac{2}{3},-\frac{1}{3},-\frac{1}{3},+\frac{2}{3},+\frac{2}{3},-\frac{1}{3},-1\right), \\
T^{\psi} & =\frac{1}{2 \sqrt{3}} \operatorname{diag}(1,1,1,1,-2,-2,0) .
\end{aligned}
$$

The normalization of $Q^{\psi}$ is fixed by the conventional electric charges of the particles. For $T$ we have used the conventional normalization for generators of the $S U(3)$ color gauge group [27]. The kinetic term in the lagrangian of the fermions is $\bar{\psi} \gamma^{\mu} D_{\mu} \psi$, where the covariant derivative of the fermion fields is

$$
D_{\mu} \psi=\partial_{\mu} \psi-i e A_{\mu}^{Q} Q^{\psi} \psi-i g A_{\mu}^{T} T^{\psi} \psi .
$$

The electromagnetic gauge coupling is $e$, and the QCD gauge coupling is $g$. The photon gauge field is $A^{Q}$, and the gluon gauge field is $A^{T}$. With the normalization of (18), $\alpha=$ $e^{2} / 4 \pi=1 / 137$, and $\alpha_{s}=g^{2} / 4 \pi \sim 1$.

\section{The 2SC condensate}

The 2SC condensate is a diquark condensate,

$$
\phi_{i j}=\left\langle\psi_{i} C \gamma_{5} \psi_{j}\right\rangle,
$$

where the indices $i$ and $j$ live in the color-flavor space of (17). The condensate only involves the red and green up and down quarks, so its color-flavor structure is

$$
\phi \propto\left(\begin{array}{rrrrrrr}
0 & 1 & 0 & 0 & 0 & 0 & 0 \\
1 & 0 & 0 & 0 & 0 & 0 & 0 \\
0 & 0 & 0 & -1 & 0 & 0 & 0 \\
0 & 0 & -1 & 0 & 0 & 0 & 0 \\
0 & 0 & 0 & 0 & 0 & 0 & 0 \\
0 & 0 & 0 & 0 & 0 & 0 & 0 \\
0 & 0 & 0 & 0 & 0 & 0 & 0
\end{array}\right) .
$$

From (18) we can see how $\phi_{i j}$, considered as a $7 \times 7$ matrix in the color-flavor space of (17), transforms under an infinitesimal electromagnetism or color rotation. Each of the quarks in the diquark feels its own color-flavor phase, so each index $i$ and $j$ is separately transformed:

$$
\begin{aligned}
& Q^{\phi} \phi=Q^{\psi} \cdot \phi+\phi \cdot Q^{\psi}, \\
& T^{\phi} \phi=T^{\psi} \cdot \phi+\phi \cdot T^{\psi},
\end{aligned}
$$


where the "." on the right hand side signifies ordinary matrix multiplication of the two $7 \times 7$ matrices, and we have used the fact that $Q^{\psi}$ and $T^{\psi}$ are both diagonal, and hence symmetric.

The lagrangian of the 2SC condensate (i.e. the G-L theory) contains the kinetic term $\left(D_{\mu} \phi\right)^{*} D^{\mu} \phi$, where the covariant derivative is

$$
D_{\mu} \phi=\partial_{\mu} \phi-i e A_{\mu}^{Q} Q^{\phi} \phi-i g A_{\mu}^{T} T^{\phi} \phi .
$$

This determines the coupling of the 2SC condensate to the gauge fields.

\section{B. The broken/unbroken basis}

When the 2SC condensate $\phi$ forms, one linear combination of $Q$ and $T$ is spontaneously broken: we will call it " $X$ ". The other remains unbroken: we will call it " $Q$ ",

$$
\begin{aligned}
& \tilde{Q}=Q+\eta_{1} T, \\
& X=-\eta_{2} Q+T .
\end{aligned}
$$

We determine $\eta_{1}$ by requiring that the $2 \mathrm{SC}$ condensate be invariant under $\tilde{Q}$ gauge transformations,

$$
\tilde{Q}^{\phi} \phi=0
$$

which implies that

$$
\eta_{1}=-\frac{1}{\sqrt{3}}
$$

It is natural to work in the $(\tilde{Q}, X)$ basis rather than the $(Q, T)$ basis, so we define new "rotated" gauge fields

$$
\begin{aligned}
& A^{\tilde{Q}}=\cos \varphi A^{Q}-\sin \varphi A^{T}, \\
& A^{X}=\sin \varphi A^{Q}+\cos \varphi A^{T},
\end{aligned}
$$

where the mixing angle $\varphi$ is analogous to the Weinberg angle in the standard model which parametrizes the mixing of the hypercharge and $W^{3}$ gauge bosons to yield the photon (analogous to $A^{\tilde{Q}}$ here) and the $Z$ (analogous to $A^{X}$ here). It is important that the mixing of the gauge fields is expressed in terms of an angle, so it maintains their normalization, so the gauge field kinetic terms for $A^{\tilde{Q}}$ and $A^{X}$ are still conventionally normalized. In the case of the generators, which we defined in (24), the overall normalization is not important, since it is absorbed in the new gauge couplings.

In the new basis, the covariant derivative of the fermions is

$$
D_{\mu} \psi=\partial_{\mu} \psi-i e^{(\tilde{Q})} A_{\mu}^{\tilde{Q}} \tilde{Q}^{\psi} \psi-i e^{(X)} A_{\mu}^{X} X^{\psi} \psi
$$

We will determine the new gauge couplings $e^{(\tilde{Q})}$ and $e^{(X)}$, and the mixing parameters $\eta_{2}$ and $\varphi$, by requiring that (28) be equivalent to (19) for all gauge field configurations. 


\section{C. $X$-charges of the particles and condensate}

Flux tubes will contain magnetic $X$-flux, so to determine the Aharonov-Bohm scattering parameter $\tilde{\beta}$ for each particle, we need to find the $X$-charge of each particle, corresponding to $q_{p}$ in (16). This follows straightforwardly from (28). We will also need to know the $X$-charge of the $2 \mathrm{SC}$ condensate, corresponding to $q_{c}$ in (16).

Requiring that (28) be equivalent to (19) for all gauge field configurations, and using (26), we find

$$
\begin{aligned}
\cos \varphi & =\frac{\sqrt{3} g}{\sqrt{e^{2}+3 g^{2}}} \\
\eta_{2} & =-\frac{e^{2}}{\sqrt{3} g^{2}}=-\sqrt{3} \tan ^{2} \varphi \\
e^{(\tilde{Q})} & =\frac{\sqrt{3} e g}{\sqrt{e^{2}+3 g^{2}}}=e \cos \varphi \\
e^{(X)} & =\frac{\sqrt{3} g^{2}}{\sqrt{e^{2}+3 g^{2}}}=g \cos \varphi .
\end{aligned}
$$

There is a new "rotated" electromagnetism, with coupling $e^{(\tilde{Q})}$, which is slightly smaller than the usual electromagnetic gauge coupling. The charges of the fermions under this gauge group are

$$
\tilde{Q}=\operatorname{diag}\left(\frac{1}{2},-\frac{1}{2},-\frac{1}{2}, \frac{1}{2}, 1,0,-1\right) .
$$

This agrees with the well-known results for the 2SC phase [6].

The action of the $X$-charge matrix on the $2 \mathrm{SC}$ condensate determines the $X$-charge $q_{c}$ of the condensate, in units of $e^{(X)}$; from (21), (24), and (29),

$$
\begin{aligned}
X \phi+\phi X & =q_{c} \phi \\
\text { where } q_{c} & =\frac{1}{\sqrt{3}}\left(1+\frac{e^{2}}{3 g^{2}}\right)=\frac{1}{\sqrt{3} \cos ^{2} \varphi} .
\end{aligned}
$$

The $X$-charge matrix of the fermions is

$$
\begin{aligned}
& X=\frac{1}{\sqrt{3}} \operatorname{diag}\left(\frac{1}{2}+2 \tan ^{2} \varphi, \quad \frac{1}{2}-\tan ^{2} \varphi,\right. \\
& \frac{1}{2}-\tan ^{2} \varphi, \quad \frac{1}{2}+2 \tan ^{2} \varphi, \\
& -1+2 \tan ^{2} \varphi,-1-\tan ^{2} \varphi, \\
& \left.-3 \tan ^{2} \varphi\right) \text {. }
\end{aligned}
$$

Dividing by $q_{c}(31)$ we find the Aharonov-Bohm $\tilde{\beta}$-factors of the fermions, in the basis defined by (17),

$$
\begin{aligned}
\tilde{\beta}^{\psi}=\operatorname{diag}( & \frac{1}{2}+\frac{3}{2} \sin ^{2} \varphi, \frac{1}{2}-\frac{3}{2} \sin ^{2} \varphi \\
& \frac{1}{2}-\frac{3}{2} \sin ^{2} \varphi, \frac{1}{2}+\frac{3}{2} \sin ^{2} \varphi \\
& \left.-1+3 \sin ^{2} \varphi, \quad-1,-3 \sin ^{2} \varphi\right) .
\end{aligned}
$$


Expanding in powers of $e^{2}$ (since $e \ll g$ ), we find

$$
\sin ^{2}(\varphi) \approx \frac{\alpha}{3 \alpha_{s}}
$$

so to lowest order in $\alpha$,

$$
\begin{aligned}
\tilde{\beta}^{\psi}=\operatorname{diag}( & \frac{1}{2}+\frac{\alpha}{2 \alpha_{s}}, \frac{1}{2}-\frac{\alpha}{2 \alpha_{s}}, \\
& \frac{1}{2}-\frac{\alpha}{2 \alpha_{s}}, \frac{1}{2}+\frac{\alpha}{2 \alpha_{s}}, \\
& \left.-1+\frac{\alpha}{\alpha_{s}}, \quad-1,-\frac{\alpha}{\alpha_{s}}\right) .
\end{aligned}
$$

We conclude that the gapped quarks have $\tilde{\beta}$ close to $\frac{1}{2}$, which means that they have nearmaximal Aharonov-Bohm interactions with an $X$-flux tube. Among the lighter (and hence more phenomenologically relevant) fermions, the $\tilde{Q}$-neutral bd has zero Aharonov-Bohm interaction with the flux tube, while the $b u$ and electron have the same Aharonov-Bohm factor

$$
\sin \left(\pi \tilde{\beta}^{b u}\right)=\sin \left(\pi \tilde{\beta}^{e}\right) \approx-\pi \frac{\alpha}{\alpha_{s}} .
$$

\section{RELAXATION VIA SCATTERING OFF FLUX TUBES}

\section{A. Relaxation time calculation}

In this section we compute the characteristic timescale for a perturbation from equilibrium to relax away due to scattering of the fermions off the color magnetic flux tubes. This relaxation time is a measure of the mean free time between collisions of the fermions with the flux tubes, so we will also refer to it as a collision time. Our calculation applies equally to electrons and the unpaired component of blue colored quarks in the 2SC phase, the key difference being $\tilde{\beta}$ factors in the cross-section. The Boltzmann kinetic equation for bluequark/electron distribution function $f(\boldsymbol{p}, t)$ is

$$
\begin{aligned}
\frac{\partial f(\boldsymbol{p}, t)}{\partial t} & =\frac{2 \pi N_{v}}{V} \int \frac{d^{3} p^{\prime}}{(2 \pi)^{3}}\left\{W\left(\boldsymbol{p} ; \boldsymbol{p}^{\prime}\right) f\left(\boldsymbol{p}^{\prime}, t\right)[1-f(\boldsymbol{p}, t)]\right. \\
& \left.-W\left(\boldsymbol{p}^{\prime} ; \boldsymbol{p}\right) f(\boldsymbol{p}, t)\left[1-f\left(\boldsymbol{p}^{\prime}, t\right)\right]\right\} \delta\left(\varepsilon(\boldsymbol{p})-\varepsilon\left(\boldsymbol{p}^{\prime}\right)\right)
\end{aligned}
$$

where $N_{v}$ is the number of flux tubes, $V$ is the volume, and $W\left(\boldsymbol{p}^{\prime} ; \boldsymbol{p}\right)$ is the transition probability between the states described by momenta $\boldsymbol{p}$ and $\boldsymbol{p}^{\prime}$. Time-reversal symmetry implies $W\left(\boldsymbol{p} ; \boldsymbol{p}^{\prime}\right)=W\left(\boldsymbol{p}^{\prime} ; \boldsymbol{p}\right)$. In equilibrium the fermion distribution function is given by the Fermi-Dirac distribution function

$$
f_{0}(\boldsymbol{p})=\frac{1}{1+\exp \left[\left(p-\mu_{i}\right) / T\right]}
$$

where $T$ is the temperature and $\mu_{i}$ is the chemical potential of blue quarks $(i=b)$ and electrons $(i=e)$. To solve the Boltzmann equation we shall apply the variational method, 
where the perturbations from equilibrium are described by variational trial functions whose functional form is dictated by the form of applied perturbation [28, 29]. The resulting transport coefficients are lower bounds on their exact values. The number of adjustable trial functions, which are used to maximize the entropy production via scattering, could be large. In the following we shall use one linear function $\phi$, in which case there is no need for variation, since the variational parameter cancels out. It should be kept in mind that the resulting transport coefficients are still lower bounds on their exact values. For small perturbations from equilibrium the Boltzmann equation can be linearized by writing $f(\boldsymbol{p}, t)=f_{0}(\boldsymbol{p})+\delta f(\boldsymbol{p}, t)$, where the (small) perturbation from the Fermi-Dirac form (38) is

$$
\delta f(\boldsymbol{p}, t)=-\frac{d f_{0}(\boldsymbol{p})}{d \varepsilon(\boldsymbol{p})} \phi(\boldsymbol{p}, t)
$$

where $\phi(\boldsymbol{p}, t)$ is the trial function. The linearized Boltzmann equation then reads

$$
\begin{aligned}
-\frac{\partial \phi(\boldsymbol{p}, t)}{\partial t} f_{0}(\boldsymbol{p})\left[1-f_{0}(\boldsymbol{p})\right] & =\frac{2 \pi N_{v}}{V} \int \frac{d^{3} p^{\prime}}{(2 \pi)^{3}}\left[\phi(\boldsymbol{p}, t)-\phi\left(\boldsymbol{p}^{\prime}, t\right)\right] \\
& \times W\left(\boldsymbol{p} ; \boldsymbol{p}^{\prime}\right) f_{0}\left(\boldsymbol{p}^{\prime}\right)\left[1-f_{0}(\boldsymbol{p})\right] \delta\left(\varepsilon(\boldsymbol{p})-\varepsilon\left(\boldsymbol{p}^{\prime}\right)\right) .
\end{aligned}
$$

To obtain this form of the kinetic equation we used the detailed balance conditions $f_{0}\left(\boldsymbol{p}^{\prime}\right)\left[1-f_{0}(\boldsymbol{p})\right]-f_{0}(\boldsymbol{p})\left[1-f_{0}\left(\boldsymbol{p}^{\prime}\right)\right]=0$, and $d f_{0}(\boldsymbol{p}) / d \varepsilon(\boldsymbol{p})=d f_{0}\left(\boldsymbol{p}^{\prime}\right) / d \varepsilon\left(\boldsymbol{p}^{\prime}\right)$. It is convenient to work with the Laplace transformed trial function

$$
\phi(\boldsymbol{p}, t)=\int d s e^{-s t} \phi(\boldsymbol{p}, s) .
$$

Upon Laplace transforming Eq. (40) we find

$$
\begin{aligned}
s \phi(\boldsymbol{p}, s) f_{0}(\boldsymbol{p})\left[1-f_{0}(\boldsymbol{p})\right] & =\frac{2 \pi N_{v}}{V} \int \frac{d^{3} p^{\prime}}{(2 \pi)^{3}}\left[\phi(\boldsymbol{p}, s)-\phi\left(\boldsymbol{p}^{\prime}, s\right)\right] \\
& \times W\left(\boldsymbol{p} ; \boldsymbol{p}^{\prime}\right) f_{0}\left(\boldsymbol{p}^{\prime}\right)\left[1-f_{0}(\boldsymbol{p})\right] \delta\left(\varepsilon(\boldsymbol{p})-\varepsilon\left(\boldsymbol{p}^{\prime}\right)\right) .
\end{aligned}
$$

To define a characteristic relaxation rate we assume that the trial function can be written as

$$
\phi(\boldsymbol{p}, s)=\phi(\boldsymbol{p}) \delta\left(s-s_{0}\right),
$$

in which case Eq. (42) becomes

$$
\begin{aligned}
s_{0} \phi(\boldsymbol{p}) f_{0}(\boldsymbol{p})\left[1-f_{0}(\boldsymbol{p})\right] & =\frac{2 \pi N_{v}}{V} \int \frac{d^{3} p^{\prime}}{(2 \pi)^{3}}\left[\phi(\boldsymbol{p})-\phi\left(\boldsymbol{p}^{\prime}\right)\right] \\
& \times W\left(\boldsymbol{p} ; \boldsymbol{p}^{\prime}\right) f_{0}\left(\boldsymbol{p}^{\prime}\right)\left[1-f_{0}(\boldsymbol{p})\right] \delta\left(\varepsilon(\boldsymbol{p})-\varepsilon\left(\boldsymbol{p}^{\prime}\right)\right),
\end{aligned}
$$

where the perturbation functions are now independent of $s$. We can identify $s_{0}$ with the relaxation rate (i.e. the inverse of the relaxation time) by comparing the computed kinetic coefficients with standard expressions for transport coefficients, e.g. , the electrical conductivity with the Drude formula.

To formulate the variational principle [30] we write Eq. (44) in the compact form

$$
X(\boldsymbol{p})=\int\left[\phi(\boldsymbol{p})-\phi\left(\boldsymbol{p}^{\prime}\right)\right] P\left(\boldsymbol{p}, \boldsymbol{p}^{\prime}\right) d^{3} p^{\prime}
$$


where $X(\boldsymbol{p})$ stands for the left-hand side of Eq. (44); the scattering operator $P\left(\boldsymbol{p}, \boldsymbol{p}^{\prime}\right)$ is easily read-off from the kernel on the right-hand side of Eq. (44). Since the factor $f_{0}(\boldsymbol{p})\left[1-f_{0}(\boldsymbol{p})\right]$ and the transition probability are positive definite, the operator $P\left(\boldsymbol{p}, \boldsymbol{p}^{\prime}\right)$ is positive definite. Further it is linear and self-adjoint (symmetric). Following ref. [30] we define an inner product

$$
\langle\phi, \psi\rangle \equiv \int \phi(\boldsymbol{p}) \psi(\boldsymbol{p}) d \boldsymbol{p}
$$

in terms of which

$$
\langle\phi, P \psi\rangle \equiv \frac{1}{2} \int d \boldsymbol{p} \int d \boldsymbol{p}^{\prime}\left[\phi(\boldsymbol{p})-\phi\left(\boldsymbol{p}^{\prime}\right)\right] P\left(\boldsymbol{p}, \boldsymbol{p}^{\prime}\right)\left[\psi(\boldsymbol{p})-\psi\left(\boldsymbol{p}^{\prime}\right)\right] .
$$

The variational principle states that the expression

$$
\langle\phi, X\rangle=\langle\phi, P \phi\rangle
$$

attains its maximum for the exact value $\phi_{\mathrm{ex}}$, which satisfies Eq. (45); for any other trial function $\phi$, that satisfies Eq. (48), $\langle\phi, P \phi\rangle \leq\left\langle\phi_{\mathrm{ex}}, P \phi_{\mathrm{ex}}\right\rangle$. Explicitly, Eq. (48) reads

$$
\begin{aligned}
s_{0} \int \frac{d^{3} p}{(2 \pi)^{3}} \phi(\boldsymbol{p}, s)^{2} f_{0}(\boldsymbol{p})\left[1-f_{0}(\boldsymbol{p})\right]= & \frac{2 \pi N_{v}}{V} \int \frac{d^{3} p}{(2 \pi)^{3}} \int \frac{d^{3} p^{\prime}}{(2 \pi)^{3}} \frac{1}{2}\left[\phi(\boldsymbol{p}, s)-\phi\left(\boldsymbol{p}^{\prime}, s\right)\right]^{2} \\
& W\left(\boldsymbol{p} ; \boldsymbol{p}^{\prime}\right) f_{0}\left(\boldsymbol{p}^{\prime}\right)\left[1-f_{0}(\boldsymbol{p})\right] \delta\left(\varepsilon(\boldsymbol{p})-\varepsilon\left(\boldsymbol{p}^{\prime}\right)\right) .
\end{aligned}
$$

It is also straightforward to check that the variation of Eq. (49) leads us back to "equation of motion" (44). From Eq. (49) we obtain the variational relaxation rate

$$
s_{0}=\frac{2 \pi N_{v}}{V \mathcal{D}} \int \frac{d^{3} p}{(2 \pi)^{3}} \int \frac{d^{3} p^{\prime}}{(2 \pi)^{3}} \frac{1}{2}\left[\phi(\boldsymbol{p})-\phi\left(\boldsymbol{p}^{\prime}\right)\right]^{2} W\left(\boldsymbol{p} ; \boldsymbol{p}^{\prime}\right) f_{0}\left(\boldsymbol{p}^{\prime}\right)\left[1-f_{0}(\boldsymbol{p})\right] \delta\left(\varepsilon(\boldsymbol{p})-\varepsilon\left(\boldsymbol{p}^{\prime}\right)\right),
$$

where

$$
\mathcal{D}=\int \frac{d^{3} p}{(2 \pi)^{3}} \phi(\boldsymbol{p})^{2} f_{0}(\boldsymbol{p})\left[1-f_{0}(\boldsymbol{p})\right]
$$

The exact relaxation rate $s \geq s_{0}$. We specify the form of the trial function appropriate to the problem at hand, which is the relaxation of uniform blue-quark/electron velocity $\boldsymbol{v}$ on a flux tube, as

$$
\phi(\boldsymbol{p})=\boldsymbol{p} \cdot \boldsymbol{v} C\left(p^{2}\right),
$$

where $C\left(p^{2}\right)$ is the scalar part of the trial function. In the following we will adopt the simple choice $C\left(p^{2}\right)=1$. The differential transition probability can be obtained from the AharonovBohm scattering cross-section, Eq. (15), and is given by (for details see Appendix A)

$$
d W=2 \pi \delta\left(\varepsilon^{\prime}-\varepsilon\right) 2 \pi \delta\left(p_{z}-p_{z}^{\prime}\right) \frac{4 L \sin ^{2}(\pi \tilde{\beta})}{\sin ^{2}(\phi / 2)} \frac{1}{2 \varepsilon V} \frac{d^{3} p^{\prime}}{(2 \pi)^{3} 2 \varepsilon^{\prime}}
$$

where the initial and final state momenta and energies, $p$ and $\varepsilon$, are unprimed and primed respectively (in this section we use $\phi$ for the scattering angle, as opposed to $\vartheta$ in Sec. IV). Here we have used the cylindrical coordinates coaxial with the flux tube to write $d^{3} p=$ $p_{\perp} d p_{\perp} d \phi d p_{z}$. Combining Eqs. (50) and (53) and carrying out the phase space integrals 
(the details are given in Appendix B) we obtain, to lowest order in the low-temperature expansion,

$$
s_{0}=\frac{p_{F i}^{3} v^{2} n_{v} T}{6 \pi^{2} \mathcal{D}} \sin ^{2}(\pi \tilde{\beta}),
$$

where $p_{F i}$ is the blue-quark/electron Fermi momentum, $n_{v}$ is the density of flux tubes. Eq. (51) with the trial function (52) can be computed in the low-temperature limit by approximating $d f(\boldsymbol{p}) / d \varepsilon(\boldsymbol{p}) \simeq \delta\left(\varepsilon(\boldsymbol{p})-\mu_{i}\right)$ to obtain $\mathcal{D}=p_{F i}^{4} v^{2} T / 6 \pi^{2}$, where $v$ is the fermion fluid velocity (52). The relaxation rate for particles of species $i$ scattering off flux tubes of area density $n_{v}$ is then given by

$$
\tau_{i f}^{-1} \equiv s_{0}=\frac{n_{v}}{p_{F i}} \sin ^{2}\left(\pi \tilde{\beta}_{i}\right)
$$

It easy to understand the final result (55). It is of the standard form for classical gases $\tau^{-1}=c n \sigma$, where $c=1$ is the speed of the particles, $n=n_{v}$ is the density of scattering centers, and $\sigma \propto \sin ^{2}(\pi \tilde{\beta}) / p_{F}$ is the cross section for Aharonov-Bohm scattering. Eq. (55) is relevant for thermal relaxation of the gapless fermion species in the 2SC phase. One of these, the blue down quark, has no A-B interaction with the flux tubes $(\tilde{\beta}=0)$. The other two, the electron and blue up quark, have identical A-B factors (36) although their Fermi momenta are different.

\section{B. Comparison with Coulomb scattering}

To find out whether scattering off flux tubes is likely to be an important source of relaxation, and hence a significant contributor to transport properties, it is useful to compare Eq. (55) with the collision time for screened Coulomb scattering via exchange of $\tilde{Q}$ photons.

The 2SC phase is a $\tilde{Q}$-conductor, with two species of gapless charged fermions: the bu quarks (with $\tilde{Q}$-charge +1 and chemical potential $\approx \mu$ ) and the electrons (with $\tilde{Q}$-charge +1 and chemical potential $\approx \mu_{e}$ ). There may also be muons, but their Fermi momentum will be much smaller. As mentioned in the introduction, the red and green quarks will be confined to bound states whose mass is expected to be of order $10 \mathrm{MeV}$ [11], so they play no role in transport at neutron star temperatures. Since $\mu>\mu_{e}$, the $b u$ quarks are more numerous than the electrons and have a larger phase space near their Fermi surface, so they will make the largest contribution to the collision time.

The Coulomb collision time depends on the in-medium photon spectrum, which will be affected by Debye screening and Landau damping arising from the presence of gapless charged excitations, dominantly the bu quarks because of their larger phase space. A simple estimate can be obtained by assuming that the dispersion relation is dominated by a plasmon pole. The plasma frequency $\omega_{p}$ is given by

$$
\omega_{p}^{2}=\frac{\tilde{\alpha} n_{q}}{\mu_{q}}=\frac{4}{3 \pi^{2}} \tilde{\alpha} \mu_{q}^{2}
$$

where $\tilde{\alpha}=e^{(\tilde{Q})^{2}} /(4 \pi)$ is the fine structure constant for the "rotated" $\tilde{Q}$ electromagnetism (29). The collision frequency is given by (see Eq. (10),(12),(18) of [31]),

$$
\tau_{q q}^{-1}=\frac{8 \zeta(3) \mu_{q}^{2}}{\pi^{3} \omega_{p}^{2}} \tilde{\alpha}^{2} T=\frac{6 \zeta(3)}{\pi^{2}} \tilde{\alpha} T
$$


where $\zeta(3)=1.202$. This result is valid for $T \ll \omega_{p}$, which is the relevant regime for neutron stars since $\mu_{q}$ is in the $400 \mathrm{MeV}$ range. Eq. (57) is analogous to Ref. [32]'s Eq. (62) for the thermal conduction timescale, with electromagnetic interactions (so their $\alpha_{s}$ is replaced by $\tilde{\alpha})$ and a different number of quark species. The quark-quark Coulomb collision frequency (57) is proportional to temperature $T$ whereas the particle-flux-tube collision frequency is independent of temperature. We can therefore define a temperature $T_{f}$ below which flux tubes dominate the relaxation of deviations from thermal equilibrium. From (57) and (55) we find

$$
T_{f}=\frac{\pi^{2}}{6 \zeta(3)} \frac{\sin ^{2}\left(\pi \beta_{b u}\right)}{\tilde{\alpha}} \frac{n_{v}}{\mu_{q}} .
$$

To make a numerical estimate we assume that the 2SC core contains the maximum flux tube density given by Eq. (11), and that $\alpha_{s} \approx 1$. Using (36) and the fact that $\tilde{\alpha} \approx \alpha$, we find

$$
T_{f} \approx\left(9 \times 10^{4} K\right)\left(\frac{B}{10^{14} \mathrm{G}}\right)\left(\frac{400 \mathrm{MeV}}{\mu_{q}}\right) .
$$

We conclude that for reasonable values of the magnetic field, only at very low temperatures is Aharonov-Bohm scattering off flux tubes likely to be an important source of thermal relaxation. However, it is important to note that the thermal relaxation timescale is not the only one that is relevant to transport. There is also the viscous relaxation rate (Ref. [32], Eq. (51)) and the momentum relaxation rate (Ref. [32], Eq. (32)) both of which have a much stronger $\left(\propto T^{5 / 3}\right)$ suppression at low temperatures. We defer a full discussion of transport in the 2SC phase to later work.

\section{FORCES ON THE FLUX TUBES}

We argued in Sec. III that even if the magnetic field in the core of the star is below the lower critical field, color magnetic flux tubes will still be produced in the transition to the 2SC phase. In this section we study the forces on those flux tubes, and estimate the timescale for their expulsion from the 2SC core. For this initial estimate we take in to account only the forces on the flux tubes within the 2SC core, or at its boundary. Depending on the nature of the material surrounding the core there may be additional forces, and these may modify the expulsion time in a way that would have to be calculated on a case-by-case basis.

The velocity of the flux tube is $\boldsymbol{v}_{L}$, the velocity of the normal fluid is $\boldsymbol{v}_{N}$, and the velocity of the 2SC condensate is $\boldsymbol{v}_{S}$. The forces we consider are mutual friction ("mf"), the non-dissipative (lifting) Magnus-Lorentz force ("ML"), the Iordanskii force ("Iord"), forces arising from zero modes ("zm"), and boundary forces ("bf") at the quark-hadronic boundary. We assume that local magneto-hydrostatic-gravitational equilibrium is established quickly after the transition to the 2SC phase, so there is no additional buoyancy force [33, 34]. We note that there may be additional forces due to density-dependence of the 2SC pairing gap [35], but we do not include these since there is as yet no reliable estimate of the density dependence. The equation of motion of a flux tube then has the form

$$
m_{V} \frac{d \boldsymbol{v}_{L}}{d t}=\boldsymbol{f}_{\mathrm{mf}}+\boldsymbol{f}_{\mathrm{ML}}+\boldsymbol{f}_{\mathrm{Iord}}+\boldsymbol{f}_{\mathrm{zm}}+\boldsymbol{f}_{\mathrm{bf}}
$$


where $m_{V}$ is the effective mass of a flux tube per unit length and $\boldsymbol{f}$ is a force per unit length. The boundary forces tend to pull the flux tube in a radial direction, expelling it from the 2SC core. This is resisted by the combination of the other forces.

In our calculations we will assume that the flux tubes are straight. A bent flux tube will feel an additional restoring force determined by its tension.

\section{A. The background $\tilde{Q}$ magnetic field}

In our calculations we will neglect the effect of the $\tilde{Q}$ magnetic field $B_{\tilde{Q}}$ that penetrates the 2SC core. Because of this field, $\tilde{Q}$-charged particles, including the bu quarks and electrons, will feel a Lorentz force. This will have a significant effect on the behavior of the normal fluid of quarks and electrons when the cyclotron frequency $\omega_{c}$ becomes larger than the inverse of the characteristic time for equilibration, which as we argued in Sec. VB, is the quark-quark collision time $\tau_{q q}(57)$. The dominant component of the fluid is the bu quarks, with $\tilde{Q}$-charge $e^{\tilde{Q}} \approx e$, and $B_{\tilde{Q}} \approx B$, so

$$
\omega_{c}=\frac{e B}{p_{F}},
$$

and we can neglect the effects of the magnetic field on transport when $\omega_{c} \tau_{q q} \ll 1$, where

$$
\omega_{c} \tau_{q q}=\frac{2 \pi^{3}}{3 \zeta(3)} \frac{1}{\sqrt{4 \pi \alpha}} \frac{B}{\mu_{q} T}=0.32\left(\frac{B}{10^{12} \mathrm{G}}\right)\left(\frac{10^{8} \mathrm{~K}}{T}\right)\left(\frac{400 \mathrm{MeV}}{\mu_{q}}\right) .
$$

We conclude that only for high magnetic fields (above $10^{12} \mathrm{G}$ ) or low temperatures (below $10^{8} \mathrm{~K}$ ) might the magnetic field affect thermal relaxation. We defer a discussion of this regime to future work.

\section{B. Mutual friction}

Mutual friction is a frictional force on a flux tube arising from its Aharonov-Bohm interaction with the normal fluid of gapless particles through which it is moving. Consider a vortex moving relative to the normal fluid with velocity $\boldsymbol{u}=\boldsymbol{v}_{L}-\boldsymbol{v}_{N}$. In the relaxation time approximation

$$
\boldsymbol{f}_{\mathrm{mf}}=\frac{\tau_{i f}^{-1}}{n_{v}} \int \frac{d^{3} p}{(2 \pi)^{3}} \boldsymbol{p} f_{0}(p, \boldsymbol{u}),
$$

where $\tau_{i f}^{-1}$ is the collision rate between fermions of species $i$ and flux tubes (55).

We will assume that the blue up quarks dominate the friction. This is reasonable because the blue down quarks have no $X$ charge and hence no Aharonov-Bohm interaction with the flux tube, and the electron Fermi momentum is smaller than that of the blue quarks. The equilibrium Fermi-Dirac thermal distribution of the quarks is

$$
f_{0}(p, \boldsymbol{u})=\left\{\exp \left[\left(\varepsilon-\mu_{i}+\boldsymbol{p} \cdot \boldsymbol{u}\right) / T\right]+1\right\}^{-1},
$$

where the $\boldsymbol{p} \cdot \boldsymbol{u}$ term is a correction due to the motion of the vortex relative to the thermal bath with velocity $\boldsymbol{u}$. We will compute the force to linear order in $\boldsymbol{u}$. The leading contribution 


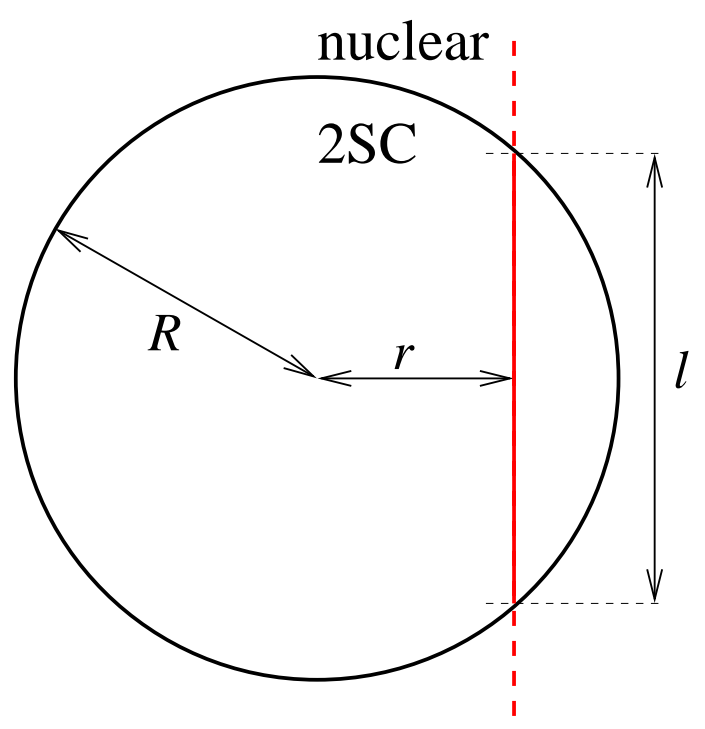

FIG. 1: A straight flux tube of length $l$ passing through a 2SC neutron star core of radius $R$, at distance $r$ from the center of the star. There is a boundary force where it reaches the edge of the 2SC core.

arises at the first order in velocity

$$
\boldsymbol{f}_{\mathrm{mf}}=\frac{\tau_{i f}^{-1}}{n_{v}} \int \frac{d^{3} p}{(2 \pi)^{3}} \boldsymbol{p}(\boldsymbol{p} \cdot \boldsymbol{u}) \frac{\partial f_{0}(\varepsilon)}{\partial \varepsilon}=\eta \boldsymbol{u} .
$$

Carrying out the integral and using (55) we obtain the mutual friction drag coefficient

$$
\eta=\frac{p_{F i} n_{i} \tau_{i f}^{-1}}{n_{v}}=n_{i} \sin ^{2}\left(\pi \tilde{\beta}_{i}\right)
$$

where $n_{i}$ is the fermion density and $\tilde{\beta}_{i}$ is their Aharonov-Bohm factor (35). As one would expect, the friction coefficient is independent of the magnetic field (i.e. the density of flux tubes). It is proportional to the fermion density, so, as noted above, the bu quark contribution will dominate the electron contribution.

\section{Boundary forces}

Next we wish to calculate the force exerted on the flux tube at the point where it reaches the interface between the 2SC quark matter core and the nuclear mantle of a neutron star (Fig. 1). When the $X$-magnetic flux tube reaches the edge of the $2 \mathrm{SC}$ core it combines with the $\tilde{Q}$ magnetic flux in the core to re-constitute the ordinary magnetic field from which it was originally formed. The form in which the flux continues through the nuclear mantle, and hence the boundary energy, may therefore be influenced by the state of the nuclear matter. In this analysis we will include only the forces arising from the contribution due to the 2SC core itself. We briefly discuss other contributions, but a proper analysis including them would have to be done in the context of a specific model of the whole neutron star and the properties of all regions within it. 
The outward force per unit length on the flux tube is (see Fig. 1)

$$
f_{b}=\frac{1}{l} \frac{d E}{d r}=\frac{r}{R^{2}-r^{2}} \varepsilon_{X}
$$

Here $E$ should be the total energy of magnetic flux inside and outside the core, but we neglect the outside contribution; $\varepsilon_{X}$ is the energy per length of $X$ flux tubes. Then from $(14)$

$$
f_{b} \approx \frac{r}{R^{2}-r^{2}} \frac{\mu_{q}^{2}}{3 \pi} \ln \kappa_{X}
$$

Taking in to account the energy of the magnetic field outside the core will reduce the right hand side of (67), and weaken the outward force on the flux tube. We now discuss the magnitude of such terms in various cases.

If the nuclear mantle is a type-II superconductor, the magnetic field penetrates the nuclear mantle in the form of Abrikosov flux tubes (dashed line in Fig. 1). From (11) we know that each $X$ flux tube will spawn 6 Abrikosov flux tubes in the nuclear matter, each of which has energy per unit length

$$
\varepsilon_{\text {nuc }}=\frac{\Phi_{0}^{2}}{4 \pi \lambda_{\text {nuc }}^{2}} \ln \kappa_{\text {nuc }} .
$$

$\Phi_{0}=\pi / e \approx 10.37$, so if we assume that the logarithmic factor is of order 1 then for $\lambda_{\text {nuc }}$ in the 50 to $100 \mathrm{fm}$ range, $\varepsilon_{\text {nuc }}$ is in the 0.2 to $0.7 \mathrm{MeV} / \mathrm{fm}$ range. This means that even when multiplied by a factor of $6, \varepsilon_{\text {nuc }}$ is small in comparison with the tension of the $X$ flux tube, which is greater than $10 \mathrm{MeV} / \mathrm{fm}(14)$, so (68) is still a good estimate of the boundary force. Of course, in a type-II nuclear mantle there may be other forces, for example if it is also a superfluid there may be entanglement of Abrikosov flux tubes with superfluid vortices, but we neglect those here because they depend on details of the nuclear mantle.

If there is no Cooper pairing of the protons then the nuclear matter is a conductor. In this case the energy gained from shortening the $X$ flux tube is counteracted by the field energy of the magnetic field it connects to in the nuclear matter mantle. The criterion for the tension of the flux tube to dominate is the same as the criterion for the magnetic field to be below its lower critical value. Since neutron star magnetic fields are well below the lower critical field for the 2SC phase, we can assume that the 2SC flux tube tension will dominate and we can use (68) again. The only complication is that conducting nuclear matter supports eddy currents which will resist any change in the magnetic field in the nuclear mantle. This may make it much harder to move the $X$-flux tubes in the 2SC core. Again, we do not attempt to include such forces that depend on details of the constitution of the nuclear mantle.

If the nuclear mantle were a type-I proton superconductor [36-39] then $X$ flux tubes in the 2SC core would connect to non-superconducting domains in the nuclear mantle [36, 39]. In this case we cannot compute the boundary force because the domain structure of the type-I proton superconductor is not known; the possible (layered, cylindrical, etc) structures in type-I superconductors essentially depend on the history of the nucleation of the superconducting phase.

\section{Magnus-Lorentz force}

The Magnus-Lorentz force is a non-dissipative force, directed orthogonally to the flux tube velocity, that arises from the superposition of the winding "flow" of the 2SC order parameter 
around the flux tube and the background flow of the charged superfluid of fermions [40-42]. (There is controversy about this in the literature; for example, Jones [33, 43] has suggested that this is cancelled by another contribution from ungapped fermions. Pending a definitive resolution of this disagreement we will use the standard form of the Magnus-Lorentz force.)

The Magnus-Lorentz force per unit length on a flux tube is

$$
\boldsymbol{f}_{\mathrm{ML}}=-\left(\boldsymbol{j}_{X} \times \hat{n} \Phi_{X}\right)
$$

where $\Phi_{X}$ is the $X$-flux through the flux tube (9), $\hat{n}$ is a unit vector pointing along the flux tube, and $\boldsymbol{j}_{X}$ is the current of $X$ charge seen by the flux tube, arising from the $X$ charge density $\rho_{X}$ of the $2 \mathrm{SC}$ condensate, moving relative to the flux tube

$$
\boldsymbol{j}_{X}=\rho_{X}\left(\boldsymbol{v}_{S}-\boldsymbol{v}_{L}\right)
$$

We can write $\rho_{X}=q_{\text {pair }} n_{s} / 2$ where $n_{s}$ is the density of quarks in the condensate. Since there are 4 quark species in the condensate, and at low temperature all fermions are part of the condensate,

$$
\begin{aligned}
\boldsymbol{f}_{\mathrm{ML}} & =-\rho\left(\boldsymbol{v}_{S}-\boldsymbol{v}_{L}\right) \times \hat{n}, \\
\rho & \equiv \rho_{X} \Phi_{X}=\pi n_{s}=\frac{4 \mu^{3}}{3 \pi} .
\end{aligned}
$$

Note that the charge of the Cooper pairs cancels in this expression.

\section{E. Iordanskii force}

The mutual friction force described above is the force on the flux tube in the longitudinal direction (i.e. parallel to its velocity relative to the normal fluid of unpaired quarks), due to Aharonov-Bohm scattering of the unpaired quarks. The Iordanskii force is the transverse component of that same force [42],

$$
\boldsymbol{f}_{\text {Iord }}=D^{\prime}\left(\boldsymbol{v}_{L}-\boldsymbol{v}_{N}\right) \times \hat{n} .
$$

The transverse Aharonov-Bohm scattering cross-section for bu quarks off the flux tube is $\sigma_{\perp}=-k^{-1} \sin \left(2 \pi \tilde{\beta}^{b u}\right)$ (Ref. [42], Eq. (64)) and, as in the case of the longitudinal AharonovBohm force, one expects the force per unit length to be proportional to the fermion density, so we expect $D^{\prime} \approx \sin \left(2 \pi \tilde{\beta}^{b u}\right) \mu_{q}^{3} \approx \alpha \mu_{q}^{3}$ (see Ref. [42], after Eq. (69)). This rough estimate is sufficient to argue that the Iordanskii force can be neglected. Basically, the AharonovBohm forces are suppressed by powers of $\alpha$ arising from the Aharonov-Bohm factor of the bu quarks (36). In the case of the Iordanskii (transverse) component, we will see that this makes it subleading relative to the Magnus-Lorentz force, which also acts perpendicular to the flux tube's velocity. In the case of the longitudinal component, there is no larger force parallel to the velocity, so the Aharonov-Bohm force is the dominant contribution to mutual friction.

\section{F. Zero-mode force}

The frictional force on a flux tube due to scattering of zero modes localized inside the flux tube off gapless fermions in the bulk [44, 45] has been calculated for proton flux tubes 
in nuclear matter [33]. At low temperatures, we expect the frictional force on a 2SC flux tube to be

$$
\boldsymbol{f}_{\|}=-\frac{C}{\omega_{0} \tau_{c}}\left(\boldsymbol{v}_{L}-\boldsymbol{v}_{N}\right)
$$

where, generalizing from nonrelativistic protons to relativistic quarks,

$$
\begin{aligned}
C & =\pi n_{q} \tanh (\Delta / 2 T) \sim \mu_{q}^{3}, \\
\omega_{0} & \sim \Delta^{2} / \mu_{q}, \\
\tau_{c} & \sim \mu_{q}^{2 / 3} T^{-5 / 3} .
\end{aligned}
$$

Eq. (75) follows from Ref. [33] Eq. (7), and the fact that 2SC pairing gap $\Delta$ is expected to be much bigger than typical neutron star temperatures. Eq. (76) follows from Ref. [33] Eq. (1), assuming, following Ref. [33], that the typical transverse momentum of the population of zero modes is of the same order as the Fermi momentum of the quarks. Eq. (77) is obtained by, as in Ref. [33], assuming that scattering involving the zero modes has the same relaxation time as quark-quark scattering in a non-superconducting medium (i.e. as if the flux tube core were infinitely large). We can then use the continuum quark-quark momentum relaxation time $\tau_{s}$ from gluon exchange in a cold quark-gluon plasma (Ref. [32], eqn (28)) as a crude estimate of the relaxation time $\tau_{c}$ for momentum transfer between bulk gapless quarks and zero modes inside the flux tube.

Comparing (74) with (65) and (36) we see that the ratio of the zero mode force to the mutual friction force is $f_{\mathrm{zm}} / f_{\mathrm{mf}} \sim\left(\omega_{0} \tau_{c} \pi^{2} \alpha^{2}\right)^{-1}$, assuming $\alpha_{s} \sim 1$. Using the estimates given above,

$$
\frac{f_{\mathrm{zm}}}{f_{\mathrm{mf}}} \sim 0.003\left(\frac{\mu_{q}}{400 \mathrm{MeV}}\right)^{1 / 3}\left(\frac{50 \mathrm{MeV}}{\Delta}\right)^{2}\left(\frac{T}{0.01 \mathrm{MeV}}\right)^{5 / 3} .
$$

We conclude that the zero mode force is likely to be negligible relative to mutual friction.

\section{G. Timescale for expulsion of flux}

We can now estimate the time scale for the expulsion of the $X$ magnetic field flux tubes from the 2SC core. As we noted above, there will be an outward force on the flux tubes at the point where they reach the nuclear mantle. The maximum force per unit length is given by (68), in which the energy costs of the magnetic field in the nuclear mantle have been neglected. The rate of outward movement of the flux tubes is given by balancing that force against frictional or pinning forces. There may be such forces arising from the nuclear matter, but we ignore them and only include the Aharonov-Bohm (mutual friction and Iordanskii) and Magnus-Lorentz forces in the quark matter. Using (60), (65), (73), (72), we can see that the steady-state value of the vortex velocity $\boldsymbol{v}_{L}$ is given by the force balance equation,

$$
\rho_{\mathrm{ML}}\left(\boldsymbol{v}_{S}-\boldsymbol{v}_{L}\right) \times \hat{n}+D^{\prime}\left(\boldsymbol{v}_{L}-\boldsymbol{v}_{N}\right) \times \hat{n}+\eta\left(\boldsymbol{v}_{L}-\boldsymbol{v}_{N}\right)+\boldsymbol{f}_{\mathrm{bf}}(r)=0,
$$

where $\boldsymbol{f}_{\mathrm{bf}}(r)$ is given by (68). We work in the reference frame that is uniformly rotating with the normal component (blue quarks and electrons) and we neglect possible small differential

rotation between the superfluid and the normal fluid, so $\boldsymbol{v}_{N}=\boldsymbol{v}_{S}=0$ in this frame. The Iordanskii and Magnus-Lorentz forces then add to give a single transverse force

$$
-\rho \boldsymbol{v}_{L} \times \hat{n}+\eta \boldsymbol{v}_{L}+\boldsymbol{f}_{\mathrm{bf}}(r)=0
$$


where $\rho=\rho_{\mathrm{ML}}-D^{\prime}$. From (72) and Sec. VIE we see that $\rho_{\mathrm{ML}} \sim \mu_{q}^{3}$ and $D^{\prime} \sim \alpha \mu_{q}^{3}$, so we can neglect the Iordanskii force and assume $\rho \approx \rho_{\mathrm{ML}}$.

We take the flux tube to lie in the $z$ direction, and we calculate its position in the $x, y$ plane using polar co-ordinates $(r, \theta)$. We want to find $\dot{r}$, the rate at which the flux tube moves outward. Solving (80) for the steady-state velocities $\dot{r}$ and $\dot{\theta}$, we find

$$
\begin{aligned}
\dot{r} & =\frac{\eta}{\eta^{2}+\rho^{2}} f_{r}(r), \\
r \dot{\theta} & =\frac{\rho}{\eta^{2}+\rho^{2}} f_{r}(r),
\end{aligned}
$$

where $f_{r}$ is the radial component of the boundary force. We note in passing that $\dot{r}$ shows a non-monotonic dependence on the friction coefficient $\eta$. As $\eta$ tends to zero one might expect the expulsion time to also tend to zero, and in the absence the Magnus-Lorentz force $(\rho=0)$ this would indeed be the case. However, in the presence of a non-zero MagnusLorentz force, the flux tube moves in an orbit around the center of the star, with the radially outward boundary force balanced by the resultant radially inward Magnus-Lorentz force.

If the flux tube starts at radius $r_{0}$ at time $t=0$ and leaves the core $(r$ reaches $R$ ) at time $t=t_{1}$, then by solving (81) we find

$$
\begin{aligned}
t_{1} & =\tau\left[2 \ln \left(\frac{R}{r_{0}}\right)+1-\frac{r_{0}^{2}}{R^{2}}\right] \\
\tau & =\frac{R^{2}}{2 \varepsilon_{X}} \frac{\eta^{2}+\rho^{2}}{\eta} .
\end{aligned}
$$

The factor in square brackets of order 1 for initial radii $r_{0}$ not too close to 0 or $R$, so the flux expulsion time for a typical flux tube is of order $\tau$. From (66) and (72), $\eta \sim \alpha^{2} \mu_{q}^{3}$ and $\rho \sim \mu_{q}^{3}$. So $\rho \gg \eta$, and using (14), (68), (36) we find

$$
\tau \approx \frac{8 \alpha_{s}^{2} \mu_{q} R^{2}}{\pi \alpha^{2} \ln \kappa_{X}} .
$$

Taking $\alpha_{s} \approx 1$

$$
\tau \approx\left(10^{10} \mathrm{yr}\right)\left(\frac{\mu_{q}}{400 \mathrm{MeV}}\right)\left(\frac{R}{1 \mathrm{~km}}\right)^{2} \frac{1}{\ln \kappa_{X}} .
$$

The timescale for $X$ flux tubes to be expelled from the 2SC core is therefore in the range of $10^{10}$ years.

\section{CONCLUSIONS}

Quark matter in the 2SC (or CFL) color-superconducting phase is a superconductor with respect to a broken " $X$ " generator that is mostly color with a small admixture of electromagnetism. We have confirmed previous calculations [7] showing that quark matter in the 2SC phase will be a type-II $X$-superconductor if the quark pairing gap is above a critical value which is well within the expected range (8). Although the ambient magnetic

field in the core of a neutron star is below the lower critical field for the formation of Abrikosov flux tubes containing $X$-magnetic flux, we argue that, when the quark matter 
cools into the 2SC phase, the process of domain formation and amalgamation is likely to leave some of the $X$ flux trapped in the form of flux tubes. The exact configuration and density of such tubes depends on details of the dynamics of the phase transition, but the density could be within an order of magnitude of the density of conventional flux tubes in proton-superconducting nuclear matter (11). Our calculations apply to 2SC quark matter in the temperature range $T_{1 S C}<T \ll T_{2 S C}$ where $T_{2 S C}$ is the critical temperature for the formation of the $2 \mathrm{SC}$ condensate, expected to be of order $10 \mathrm{MeV}\left(10^{11} \mathrm{~K}\right)$, and $T_{1 S C}$ is the critical temperature for self pairing of the blue quarks, which could be as low as $1 \mathrm{eV}\left(10^{4} \mathrm{~K}\right)$.

The 2SC phase contains three species of gapless fermions: two quarks ("blue up" and "blue down") and the electron. These are expected to dominate its transport properties. We do not discuss strange quarks, but our analysis is also applicable to phases with strange quarks present, as long as their pairing pattern does not break the $\tilde{Q}$ gauge symmetry. Muons may also be present, but, like strange quarks, their higher mass gives them a lower Fermi momentum so they make a subleading contribution to the phenomena discussed here. We have calculated the Aharonov-Bohm scattering cross-section of gapless fermions with the $X$ flux tubes (15), (36), and the associated collision (or relaxation) rate (55). A comparison with the collision time for Coulomb quark-quark scattering indicates that only at very low temperatures $\left(T \lesssim 10^{5} \mathrm{~K}\right.$ or $\left.10 \mathrm{eV}\right)$ will the flux tubes dominate over thermal relaxation via Coulomb scattering. However, we defer a detailed calculation of the transport properties, including Coulomb and $X$-boson-mediated interactions, to future work.

Because the ambient magnetic field in a neutron star is below the lower critical field required to force $X$-flux tubes into 2SC quark matter, the trapped flux tubes will feel a boundary force pulling them outwards. We calculated this force for the case where the energy of the magnetic field outside the core can be neglected relative to the energy of flux tube. This force will be balanced by the drag force ("mutual friction") on the moving flux tube due to its Aharonov-Bohm interaction with the thermal population of gapless quarks and electrons (66), and also by the Magnus-Lorentz force (72). On this basis, we estimate that the timescale for the expulsion of $X$ flux tubes from a $2 \mathrm{SC}$ core (84), is of order $10^{10}$ years.

The work described here offers many directions for future development.

(1) To get a full picture of the transport properties of 2SC quark matter one must calculate the relaxation rates associated with processes that do not include flux tubes, such as $\tilde{Q}$-Coulomb and $X$-boson-mediated interactions between gapless fermions.

(2) We studied the regime where the cyclotron frequency is smaller than the thermal relaxation time of the unpaired quarks (see Sec. VIA). It would be valuable to extend our analysis to higher magnetic fields and/or lower temperatures where the cyclotron frequency cannot be neglected.

(3) It is important to resolve the disagreement in the literature over whether the MagnusLorentz force on flux tubes is cancelled by forces arising from the neutralizing background (see Sec. VID). This is necessary for understanding flux expulsion from superconducting nuclear matter as well as more exotic flux tubes such as the ones that we described here.

(4) We assumed that the $X$-flux tubes are stable, or at least have a lifetime that is long enough for them to play a role in transport. However there is no topological guarantee of their stability, and it is necessary to perform a calculation of their energetics, analogous to that of [13], and to investigate bound states on the string, which if present can enhance their stability [14].

(5) We focussed on the 2SC phase, but other phases may support flux tubes. The CFL 
phase, which is the ground state of 3-flavor quark matter at asymptotically high densities, also has a gauge symmetry breaking pattern which resolves an external magnetic field in to an unbroken $\tilde{Q}$ part, and a broken $X$ part which could be carried in flux tubes [46]. In this case also there is no topological guarantee of stability, and an analysis of the energetic stability is required. The CFL phase also features semi-superfluid vortices with non-zero magnetization [7, 47-50]. Since the CFL phase has no gapless charged excitations the associated phenomenology is likely to be quite different. In the CFL-K0 phase there are charged kaon modes that can have an energy gap well below the pairing gap, so, if they have non-zero Aharonov-Bohm $\tilde{\beta}$ factors, their scattering off flux tubes might be important.

(6) We treated the thickness of the flux tubes as negligible, so scattering off them is dominated by the Aharonov-Bohm effect. In fact the thickness of the flux tube is comparable to the inverse Fermi momentum of the quarks (see (12)) and there will be finite-size corrections to our results. Calculating them would require explicit construction of the radial profile of the flux tube.

(7) Some quark matter phases break the $\tilde{Q}$ gauge symmetry. These include the 2SC phase at $T<T_{1 S C}$, and many other phases such as the color-spin-locked phase [16, 51]. It is interesting to ask what happens to magnetic flux in such cases: is the $\tilde{Q}$-superconductivity always type-I? (One suspects it may be because the gaps are usually small.) Will the dynamics of the phase transition lead to trapped normal regions, and what is the timescale for their expulsion from the star? Could these phases retain $X$-flux tubes even after $\tilde{Q}$ flux has been expelled? If $X$-flux tubes existed in a CFL core, for example they might experience the same sort of entanglement with superfluid vortices as is predicted in nuclear matter.

(8) Neutron stars probably have layers of different phases. For a proper treatment of the dynamics of magnetic flux one would have to analyse how magnetic flux was connected between layers and pinned within layers, and the consequent additional forces on the color magnetic flux tube in a 2SC core. For instance, in a conducting nuclear mantle there would be eddy-current pinning of the magnetic flux; in a type-II superconducting and superfluid mantle there would be entanglement of nuclear Abrikosov flux tubes with superfluid vortices; and so on. There is also the possibility of different quark matter phases, such as an inner CFL core, inside the 2SC region. If it turned out that additional forces arising from these other regions of the star acted so as to allow expulsion of the flux tubes on a shorter timescale then this would have interesting astrophysical ramifications, such as a change of the magnetic moment of the star over this period of time. If the core contained a phase where $X$-flux tubes were entangled with superfluid vortices (as mentioned for the CFL phase above) then the rotational dynamics could also be affected. Observationally, this could provide a new mechanism for glitches in neutron stars, since vortex-interface pinning force, derived above, may prevent a continuous flow of rotational vortices in the superfluid phases, in a manner analogous to vortex pinning in the crust [52] and the hadronic core-solid crust interface [53]. Other dynamical manifestations, such as, for example, the recently studied shear modes $[54,55]$ in the superfluid core and the post-jump relaxations (see Ref. [22] and references therein) will be affected as well.

\section{Acknowledgements}

We thank Xu-Guang Huang, Kazunori Itakura, Naoki Itoh, Peter Jones, Muneto Nitta, Dirk Rischke, Karen Shahabasyan for their comments. This research was supported in part by the Offices of Nuclear Physics and High Energy Physics of the U.S. Department of 
Energy under contracts \#DE-FG02-91ER40628, \#DE-FG02-05ER41375, and the Deutsche Forschungsgemeinschaft (Grant SE 1836/1-1).

\section{Appendix A: Relating the scattering amplitude to Aharonov-Bohm cross-section}

The differential scattering probability is given by [56]

$$
d W=2 \pi \delta\left(\varepsilon^{\prime}-\varepsilon\right) 2 \pi \delta\left(p_{z}-p_{z}^{\prime}\right)\left|M_{f i}\right|^{2} \frac{1}{2 \varepsilon V} \prod_{a} \frac{d^{3} p_{a}^{\prime}}{(2 \pi)^{3} 2 \varepsilon_{a}^{\prime}} .
$$

It is assumed that a particle scatters off a heavy center; the momentum conserving delta function reflects the fact that there is no scattering along the vortex (i.e., in the $z$ direction). The quantities referring to the final states are primed, those to the initial state are unprimed. The $a$ products is over all the final state particle phase space. The differential scattering cross section is

$$
d \sigma=\frac{d W}{j}, \quad j=\frac{V}{v}=\frac{\varepsilon V}{|\boldsymbol{p}|},
$$

here $j$ is the current, $v$ the velocity, and $V$ the volume. Substituting (A2) in Eq. (A1) we obtain for the scattering cross-section

$$
d \sigma=j^{-1} 2 \pi \delta\left(\varepsilon^{\prime}-\varepsilon\right) 2 \pi \delta\left(p_{z}-p_{z}^{\prime}\right)\left|M_{f i}\right|^{2} \frac{1}{2 \varepsilon V} \prod_{a} \frac{d^{3} p_{a}^{\prime}}{(2 \pi)^{3} 2 \varepsilon_{a}^{\prime}} .
$$

The differential scattering cross section is obtained on writing $d^{3} p^{\prime}=d p_{\perp}^{\prime} p_{\perp}^{\prime} d \phi^{\prime} d z^{\prime}$ (we restrict in the following the $a$ product to one particle, since we consider elastic scattering and there is the same blue quark or electron in the final state). Thus,

$$
\frac{d \sigma}{d \phi^{\prime}}=j^{-1} 2 \pi \delta\left(\varepsilon^{\prime}-\varepsilon\right) 2 \pi \delta\left(p_{z}-p_{z}^{\prime}\right)\left|M_{f i}\right|^{2} \frac{1}{2 \varepsilon V} \frac{d p_{\perp}^{\prime} p_{\perp}^{\prime} d p_{z}^{\prime}}{(2 \pi)^{3} 2 \varepsilon^{\prime}} .
$$

The final state energy is $\varepsilon^{\prime}=\sqrt{p_{\perp}^{\prime 2}+p_{z}^{\prime 2}}$, therefore $\varepsilon^{\prime} d \varepsilon^{\prime}=p_{\perp}^{\prime} d p_{\perp}^{\prime}$. After integrating by means of delta-functions we obtain

$$
\frac{d \sigma}{d \phi^{\prime}}=j^{-1}\left|M_{f i}\right|^{2} \frac{1}{8 \pi \varepsilon V}
$$

The current density is given by $j=v_{\perp} / L^{2}$, where $v_{\perp}=\left|p_{\perp}\right| / \varepsilon[56]$. Comparing (A4) with Eq. (15) we obtain

$$
\left|M_{f i}\right|^{2}=\frac{4 L \sin ^{2}(\pi \tilde{\beta})}{\sin ^{2}(\phi / 2)} .
$$

Finally, substituting this result in Eq. (A1) we arrive at Eq. (53) of the main text.

\section{Appendix B: Phase space integrals}

Upon substituting the transition probability in the rate Eq. (49) and introducing momentum transfer $\boldsymbol{k}=\boldsymbol{p}^{\prime}-\boldsymbol{p}$ we find

$$
s_{0}=\frac{4 \pi}{\hbar} \frac{n_{v}}{\mathcal{D}} \sin ^{2}(\pi \tilde{\beta}) \int \frac{d^{3} k}{(2 \pi)^{2}}[\boldsymbol{k} \cdot \boldsymbol{v}]^{2} \delta\left(k_{z}\right) I_{p}
$$


where we replaced $N_{v} L / V=n_{v}$, which is the density of flux tubes per unit area. The integral $I_{p}$ is defined as

$$
I_{p}=\int \frac{d^{3} p}{(2 \pi)^{3}} f_{0}(\boldsymbol{p})\left[1-f_{0}(\boldsymbol{p})\right] \delta\left(\varepsilon(\boldsymbol{p})-\varepsilon\left(\boldsymbol{k}_{\perp}-\boldsymbol{p}\right)\right) \frac{1}{\sin ^{2}(\phi / 2)} \frac{1}{4 \varepsilon(\boldsymbol{p})^{2}} .
$$

The form of the scattering probability suggest that the phase space integral over $d^{3} p$ is convenient to carry out in the cylindrical coordinates by writing $d^{3} p=p_{\perp} d p_{\perp} d \phi d p_{z}$ and

$$
I_{p}=\frac{1}{4(2 \pi)^{3}} \int d p_{\perp} p_{\perp} d \phi \frac{1}{\sin ^{2}(\phi / 2)} \int d p_{z} f_{0}(\boldsymbol{p})\left[1-f_{0}(\boldsymbol{p})\right] \frac{1}{\varepsilon(\boldsymbol{p})^{2}} \delta\left(\varepsilon(\boldsymbol{p})-\varepsilon\left(\boldsymbol{p}-\boldsymbol{k}_{\perp}\right)\right) .
$$

To do the inner integral note that in the low-temperature limit

$$
f_{0}(\boldsymbol{p})\left[1-f_{0}(\boldsymbol{p})\right]=T \frac{d f_{0}(\boldsymbol{p})}{d \varepsilon(\boldsymbol{p})} \simeq T \frac{p_{F}}{\sqrt{p_{F}^{2}-p_{\perp}^{2}}} \delta\left(p_{z}-p_{z}^{0}\right)
$$

where in the last step we used cylindrical polar coordinates with $p_{z}^{0}=\sqrt{\varepsilon_{F}^{2}-p_{\perp}^{2}}$. Carrying out the $p_{z}$-integration we obtain

$$
I_{p}=-\frac{T}{4(2 \pi)^{3}} \int \frac{d p_{\perp} p_{\perp}}{p_{F} \sqrt{p_{F}^{2}-p_{\perp}^{2}}} \theta\left(\sqrt{p_{F}^{2}-p_{\perp}^{2}}\right) I_{\phi},
$$

where

$$
I_{\phi}=\int\left[\frac{d \phi}{\sin ^{2}(\phi / 2)}\right] \delta\left(\varepsilon_{F}-\sqrt{p_{F}^{2}+k_{\perp}^{2}-2 \boldsymbol{p}_{\perp} \cdot \boldsymbol{k}_{\perp}}\right) .
$$

We next specify the geometry of the scattering, by assuming that the vortex is along z-axis, vector $p$ is directed along the $x$ axis, and the scattering is in the $x$-y plane. If we denote the angle formed by the vectors $\boldsymbol{p}_{\perp}$ and $\boldsymbol{k}_{\perp}$ by $\chi$ then, $\cos \chi=\sin \phi / 2$. We next note that identically $d \phi=-2 d \sin (\phi / 2) / \cos (\phi / 2)$ and define

$$
\sin \frac{\phi_{0}}{2}=\frac{k_{\perp}}{2 p_{\perp}} .
$$

The integral becomes

$$
\begin{aligned}
I_{\phi} & =-2 \frac{p_{F}}{p_{\perp} k_{\perp}} \int_{-1}^{1} \frac{d \sin (\phi / 2)}{\cos (\phi / 2)}\left[\frac{1}{\sin ^{2}(\phi / 2)}\right] \delta\left(\sin \phi / 2-\sin \phi_{0} / 2\right) \\
& =-\frac{16 p_{F} p_{\perp}^{2}}{k_{\perp}^{3}} \frac{1}{\sqrt{4 p_{\perp}^{2}-k_{\perp}^{2}}} \theta\left(1-\frac{k_{\perp}}{2 p_{\perp}}\right) .
\end{aligned}
$$

The integration limits were chosen for convenience $[-1 ; 1]$; the integral is then multiplied by a factor 2 to account for full 360 degree angle range. Inserting these results in Eq. (B5) we obtain

$$
I_{p}=\frac{2 T}{(2 \pi)^{3} k_{\perp}^{3}} \int \frac{d p_{\perp} p_{\perp}^{3}}{\sqrt{p_{F}^{2}-p_{\perp}^{2}}} \frac{1}{\sqrt{p_{\perp}^{2}-\frac{k_{\perp}^{2}}{4}}} \theta\left(1-\frac{k_{\perp}}{2 p_{\perp}}\right) \theta\left(\sqrt{p_{F}^{2}-p_{\perp}^{2}}\right)
$$


The integration is carried out using

$$
\int_{a}^{b} d x \frac{x^{3}}{\sqrt{b^{2}-x^{2}} \sqrt{x^{2}-a^{2}}}=\frac{\pi}{4}\left(b^{2}+a^{2}\right),
$$

to obtain the final expression for the momentum integral

$$
I_{p}=\frac{T}{16 \pi^{2} k_{\perp}^{3}} \theta\left(1-\frac{k_{\perp}}{2 p_{F}}\right)\left(p_{F}^{2}+\frac{k_{\perp}^{2}}{4}\right) .
$$

Next we substitute this result in Eq. (B1) and obtain

$$
s_{0}=\frac{n_{v} v^{2} T}{4 \pi \hbar \mathcal{D}} \sin ^{2}(\pi \tilde{\beta}) \int_{0}^{2 p_{F}} \frac{d k_{\perp}}{(2 \pi)^{2}}\left(p_{F}^{2}+\frac{k_{\perp}^{2}}{4}\right) \int_{0}^{2 \pi} d \phi(\cos \phi)^{2} .
$$

The angular integral is equal $\pi$, the remaining integral is $(8 / 3) p_{F}^{3}$, and we arrive at Eq. (54) of the main text.

[1] X.-G. Huang, M. Huang, D. H. Rischke, and A. Sedrakian, Anisotropic Hydrodynamics, Bulk Viscosities and R-Modes of Strange Quark Stars with Strong Magnetic Fields, Phys. Rev. D81 (2010) 045015, [arXiv:0910.3633].

[2] E. J. Ferrer, V. de la Incera, and C. Manuel, Color-superconducting gap in the presence of a magnetic field, Nucl. Phys. B747 (2006) 88-112, [hep-ph/0603233].

[3] K. Fukushima and H. J. Warringa, Color superconducting matter in a magnetic field, Phys. Rev. Lett. 100 (2008) 032007, [arXiv:0707.3785].

[4] D. P. Menezes, M. Benghi Pinto, S. S. Avancini, A. Perez Martinez, and C. Providencia, Quark matter under strong magnetic fields in the Nambu-Jona-Lasinio Model, Phys. Rev. C79 (2009) 035807, [arXiv:0811.3361].

[5] G. Baym, C. Pethick, and D. Pines, Superfluidity in Neutron Stars, Nature (London) 224 (Nov., 1969) 673-674.

[6] M. G. Alford, A. Schmitt, K. Rajagopal, and T. Schafer, Color superconductivity in dense quark matter, Rev. Mod. Phys. 80 (2008) 1455-1515, [arXiv:0709.4635].

[7] K. Iida and G. Baym, Superfluid phases of quark matter. III: Supercurrents and vortices, Phys. Rev. D66 (2002) 014015, [hep-ph/0204124].

[8] I. Giannakis and H.-c. Ren, The Ginzburg-Landau theory and the surface energy of a colour superconductor, Nucl. Phys. B669 (2003) 462-478, [hep-ph/0305235].

[9] M. G. Alford, K. Rajagopal, and F. Wilczek, QCD at finite baryon density: Nucleon droplets and color superconductivity, Phys. Lett. B422 (1998) 247-256, [hep-ph/9711395].

[10] M. G. Alford, J. Berges, and K. Rajagopal, Magnetic fields within color superconducting neutron star cores, Nucl. Phys. B571 (2000) 269-284, [hep-ph/9910254].

[11] D. H. Rischke, D. T. Son, and M. A. Stephanov, Asymptotic deconfinement in high-density QCD, Phys. Rev. Lett. 87 (2001) 062001, [hep-ph/0011379].

[12] T. Vachaspati, Vortex solutions in the Weinberg-Salam model, Phys. Rev. Lett. 68 (1992) 1977-1980.

[13] M. James, L. Perivolaropoulos, and T. Vachaspati, Detailed stability analysis of electroweak strings, Nucl. Phys. B395 (1993) 534-546, [hep-ph/9212301]. 
[14] T. Vachaspati and R. Watkins, Bound states can stabilize electroweak strings, Phys. Lett. B318 (1993) 163-168, [hep-ph/9211284].

[15] M. G. Alford, J. A. Bowers, J. M. Cheyne, and G. A. Cowan, Single color and single flavor color superconductivity, Phys. Rev. D67 (2003) 054018, [hep-ph/0210106].

[16] T. Schafer, Quark hadron continuity in QCD with one flavor, Phys. Rev. D62 (2000) 094007, [hep-ph/0006034].

[17] D. Bailin and A. Love, Superfluidity and Superconductivity in Relativistic Fermion Systems, Phys. Rept. 107 (1984) 325-385.

[18] W. E. Brown, J. T. Liu, and H.-c. Ren, On the perturbative nature of color superconductivity, Phys. Rev. D61 (2000) 114012, [hep-ph/9908248].

[19] W. E. Brown, J. T. Liu, and H.-c. Ren, The transition temperature to the superconducting phase of QCD at high baryon density, Phys. Rev. D62 (2000) 054016, [hep-ph/9912409].

[20] D. J. Dean and M. Hjorth-Jensen, Pairing in nuclear systems: from neutron stars to finite nuclei, Rev. Mod. Phys. 75 (2003) 607-656, [nucl-th/0210033].

[21] H. Muther and W. H. Dickhoff, Pairing properties of nucleonic matter employing dressed nucleons, Phys. Rev. C72 (2005) 054313, [nucl-th/0508035].

[22] A. Sedrakian and J. W. Clark, Nuclear Superconductivity in Compact Stars: BCS Theory and Beyond, nucl-th/0607028.

[23] D. M. Sedrakian, D. Blaschke, K. M. Shahabasyan, and D. N. Voskresensky, Meissner Effect for "Color" Superconducting Quark Matter, Astrophysics 44 (July, 2001) 359-368, [hep-ph/00].

[24] D. Blaschke and D. Sedrakian, Ginzburg-Landau equations for superconducting quark matter in neutron stars, nucl-th/0006038.

[25] M. Tinkham, Introduction to Superconductivity. McGraw-Hill, 2nd ed., 1996.

[26] M. G. Alford and F. Wilczek, Aharonov-Bohm Interaction of Cosmic Strings with Matter, Phys. Rev. Lett. 62 (1989) 1071.

[27] J. F. Donoghue, E. Golowich, and B. R. Holstein, Dynamics of the standard model, vol. 2 of Camb. Monogr. Part. Phys. Nucl. Phys. Cosmol. Cambridge University Press, 1st ed., 1992.

[28] E. Flowers and N. Itoh, Transport properties of dense matter, Astrophys. J. 206 (May, 1976) 218-242.

[29] E. Flowers and N. Itoh, Transport properties of dense matter. II, Astrophys. J. 230 (June, 1979) 847-858.

[30] J. M. Ziman, Electrons and Phonons. Claredon Press, Oxford, 1960.

[31] P. S. Shternin and D. G. Yakovlev, Electron thermal conductivity owing to collisions between degenerate electrons, Phys. Rev. D74 (2006) 043004, [astro-ph/0608371].

[32] H. Heiselberg and C. J. Pethick, Transport and relaxation in degenerate quark plasmas, Phys. Rev. D48 (1993) 2916-2928.

[33] P. B. Jones, Fermion zero-mode influence on neutron-star magnetic field evolution, Mon. Not. R. Astron. Soc.397 (Aug., 2009) 1027-1031, [arXiv: 0902.0478].

[34] A. G. Muslimov and A. I. Tsygan, Neutron Star Superconductivity and Superfluidity and the Decay of Pulsar Magnetic Fields, Soviet Astronomy Letters 11 (Mar., 1985) 80-83.

[35] S. D. H. Hsu, Neutron Star Vortex Dynamics and Magnetic Field Decay: Implications for High Density Nuclear Matter, Phys. Lett. B469 (1999) 161-165, [nucl-th/9903039].

[36] A. Sedrakian, Type-I superconductivity and neutron star precession, Phys. Rev. D71 (2005) 083003, [astro-ph/0408467].

[37] M. Alford, G. Good, and S. Reddy, Isospin asymmetry and type-I pairing in neutron star 
matter, Phys. Rev. C72 (2005) 055801, [nucl-th/0505025].

[38] M. G. Alford and G. Good, Flux tubes and the type-I/type-II transition in a superconductor coupled to a superfluid, Phys. Rev. B78 (2008) 024510, [arXiv:0712.1810].

[39] J. Charbonneau and A. Zhitnitsky, A Novel Mechanism for Type-I Superconductivity in Neutron Stars, Phys. Rev. C76 (2007) 015801, [astro-ph/0701308].

[40] J. Bardeen and M. J. Stephen, Theory of the motion of vortices in superconductors, Phys. Rev. 140 (Nov, 1965) A1197-A1207.

[41] G. Mendell, Superfluid Hydrodynamics in Rotating Neutron Stars. II. Dissipative Effects, Astrophys. J. 380 (Oct., 1991) 530.

[42] E. B. Sonin, Magnus force in superfluids and superconductors, Phys. Rev. B 55 (Jan, 1997) 485-501.

[43] P. B. Jones, Neutron superfluid spin-down and magnetic field decay in pulsars, Mon. Not. R. Astron. Soc.253 (Nov., 1991) 279-286.

[44] G. E. Volovik, Comment on "Transverse force on a quantized vortex in a superfluid", Phys. Rev. Lett. 77 (Nov, 1996) 4687.

[45] N. B. Kopnin, Vortex dynamics and mutual friction in superconductors and Fermi superfluids, Rep. Prog. Phys. 65 (2002) 1633-1678.

[46] K. Iida, Magnetic vortex in color-flavor locked quark matter, Phys. Rev. D71 (2005) 054011, [hep-ph/0412426].

[47] A. P. Balachandran, S. Digal, and T. Matsuura, Semi-superfluid strings in high density QCD, Phys. Rev. D73 (2006) 074009, [hep-ph/0509276].

[48] M. Eto, E. Nakano, and M. Nitta, Effective world-sheet theory of color magnetic flux tubes in dense QCD, Phys. Rev. D80 (2009) 125011, [arXiv:0908.4470].

[49] M. Eto, E. Nakano, and M. Nitta, Non-Abelian Global Vortices, Nucl. Phys. B821 (2009) 129-150, [arXiv:0903.1528].

[50] D. M. Sedrakian, K. M. Shahabasyan, D. Blaschke, and K. M. Shahabasyan, Vortex structure of a neutron star with CFL quark core, Astrophys. 51 (2008) 544-558.

[51] A. Schmitt, Q. Wang, and D. H. Rischke, Electromagnetic Meissner effect in spin-one color superconductors, Phys. Rev. Lett. 91 (2003) 242301, [nucl-th/0301090].

[52] P. W. Anderson and N. Itoh, Pulsar glitches and restlessness as a hard superfluidity phenomenon, Nature 256 (1975) 25-27.

[53] A. Sedrakian and J. M. Cordes, Vortex-interface interactions and generation of glitches in pulsars, Mon. Not. Roy. Astron. Soc. 307 (1999) 365, [astro-ph/9806042].

[54] J. Noronha and A. Sedrakian, Tkachenko modes as sources of quasiperiodic pulsar spin variations, Phys. Rev. D77 (2008) 023008, [arXiv:0708.2876].

[55] M. K. Shahabasyan, Vortex lattice oscillations in rotating neutron stars with quark 'CFL' cores, Astrophys. 52 (2009) 151-155.

[56] V. B. Berestetskii, E. M. Lifshitz, and L. P. Pitaevskii, Course of Theoretical Physics, Vol.4: Quantum Electrodynamics. Butterworth-Heinemann, New York, 2nd ed., 1982. 